Pacific

Journal of

Mathematics

\title{
ON THE DIMENSION OF THE SET OF EXTREMAL DISCS FOR A CR MANIFOLD OF CODIMENSION TWO
}

ALberto ScALARI

Volume $223 \quad$ No. 2

February 2006 


\title{
ON THE DIMENSION OF THE SET OF EXTREMAL DISCS FOR A CR MANIFOLD OF CODIMENSION TWO
}

\author{
Alberto ScAlari
}

\begin{abstract}
We show that, in codimension higher than one, perturbing a quadric may result in raising the dimension of the set of extremal discs.
\end{abstract}

\section{Introduction}

Lempert [1981] introduced extremal discs for a convex domain in $\mathbb{C}^{n}$, a very productive notion which has had many applications. Tumanov [2001] introduced a local theory of extremal discs attached to a real strictly pseudoconvex manifold of higher codimension. For a hypersurface, his discs coincide with those of Lempert. An important feature of extremal discs for a hypersurface is that of stability under small perturbations. In particular, the dimension of the set of extremal discs depends only on the dimension of the ambient space. We show that this property is significantly violated in higher codimension.

Let $(z, w) \in \mathbb{C}^{4}$, with $z=\left(z_{1}, z_{2}\right)$ and $w=\left(w_{1}, w_{2}\right)$, where $z_{1}=x_{1}+i y_{1}$ and $z_{2}=x_{2}+i y_{2}$. Let $\sigma \geq 0$ and let $Q_{\sigma}$ be the manifold defined by

$$
x_{1}=\left|w_{1}\right|^{2}+\sigma \operatorname{Re}\left(w_{2} \bar{w}_{1}^{2}\right), \quad x_{2}=\left|w_{2}\right|^{2} .
$$

Theorem 1. Extremal discs attached to $Q_{0}$ depend on 14 parameters. For $\sigma>0$ there is a family of extremal discs attached to $Q_{\sigma}$ which depend on 15 parameters.

This result has led Sukhov and Tumanov [2001] to the notion of an extended indicatrix, the higher-codimensional analogue of Lempert's indicatrix. They make an adjustment to address the above instability.

\section{Main Result}

Consider a rigid, analytic, strictly pseudoconvex manifold $M$ in $\mathbb{C}^{4}$, of codimension two. We choose a neighborhood $U$ of zero in $\mathbb{C}^{4}$, inside which $M$ is described by

$$
x_{i}=h_{i}(w)=\left|w_{i}\right|^{2}+g_{i}(w)
$$

MSC2000: primary 32V20; secondary $32 \mathrm{H} 25$.

Keywords: extremal discs, CR manifolds. 
for $i=1,2$, with $g_{i}$ real analytic, and vanishing at 0 of order greater than two. Let $\Delta$ be the standard unit disc, with boundary $b \Delta$ and closure $\bar{\Delta}$. An analytic disc attached to $M$ is a map $f: \bar{\Delta} \rightarrow \mathbb{C}^{n}$ that is holomorphic on $\Delta$ and continuous up to the boundary $b \Delta$, such that $f(b \Delta) \subset M$ (see [Tumanov 2001]).

In what follows, we consider only the set $A^{M}$ of analytic discs attached to $M$ and contained in $U$. A disc $f$ in $A^{M}$ has the form $f(\zeta)=(z(\zeta), w(\zeta))$, where $x(\zeta)=h(w(\zeta))$ for $\zeta \in \partial \Delta$ and $y(\zeta)$ is related to $x(\zeta)$ by the Hilbert transform

$$
x(\zeta)=-T y(\zeta)+x(1)
$$

(see [Boggess 1991] or [Tumanov 2001]). We also consider the set

$$
A^{M}\left(w_{0}^{\prime}\right)=\left\{(z(\zeta), w(\zeta)) \in A^{M}: w(0)=0, y(0)=0, w^{\prime}(0)=w_{0}^{\prime}\right\} .
$$

Let $N^{*} M$ denote the conormal bundle to $M$. The following definitions are from [Lempert 1981] and [Tumanov 2001].

Definition 2. An analytic disc attached to $M, f: \Delta \rightarrow \mathbb{C}^{4}$ is stationary if there exists a nonzero smooth section $f^{*}(\zeta)$ of $N^{*} M$, defined for $|\zeta|=1$, that is, $f^{*}(\zeta) \in N_{f(\zeta)}^{*} M$ such that $\zeta f^{*}(\zeta)$ extends to a continuous function on $\bar{\Delta}$ that is holomorphic on $\Delta$.

The map $f^{*}$ is called a lift of $f$. For two vectors $a, b \in \mathbb{C}^{n}$, we set $\langle a, b\rangle=\Sigma a_{j} b_{j}$.

Definition 3. An analytic disc $f: \bar{\Delta} \rightarrow \mathbb{C}^{4}$ attached to $M$ is extremal if there exists a vector $a \in \mathbb{C}^{4}$ such that $\operatorname{Re}\left\langle\bar{a}, g^{\prime}(0)-f^{\prime}(0)\right\rangle>0$ for every analytic disc $g: \bar{\Delta} \rightarrow \mathbb{C}^{4}$ attached to $M$ where $g \neq f$ and $g(0)=f(0)$.

Let $Q$ be the quadric in $\mathbb{C}^{2}$ defined by the equation $x_{1}=\left|w_{1}\right|^{2}$. Then $Q_{0}=Q \times Q$. An extremal disc $f$ in $A^{Q_{0}}$ has components $f_{1}, f_{2}$ that are extremal discs attached to $Q$ and each depend on 7 parameters (see [Lempert 1981]). As a consequence, $f$ depends on 14 parameters, as stated in Theorem 1 . When we perturb $Q_{0}$, we lose the structure of product and we can not apply a similar argument.

We recall Tumanov's existence theorem for stationary discs in our particular case. Let $\rho$ be the vector-valued function with components $x_{i}-h_{i}(w)$.

Proposition 4. For every $\epsilon>0$ there exists $\delta>0$ such that, for every $\lambda, w_{0}, w_{0}^{\prime} \in \mathbb{C}^{2}$ and every $c, y_{0} \in \mathbb{R}^{2}$ satisfying the conditions $\left|w_{0}\right|<\delta,\left|y_{0}\right|<\delta,\left|w_{0}^{\prime}\right|<\delta$, and

(3) $\operatorname{Re}\left(\lambda_{i} \zeta+c_{i}\right)>\epsilon(|\lambda|+|c|) \quad$ for $i=1,2$ and all $\zeta \in \mathbb{C}$ such that $|\zeta|=1$,

there exists a unique stationary disc

$$
\zeta \mapsto f(\zeta)=(z(\zeta), w(\zeta))
$$

attached to $M$, with $w(0)=w_{0}, w^{\prime}(0)=w_{0}^{\prime}, y(0)=y_{0}$ and $\left.f^{*}\right|_{b \Delta}=\operatorname{Re}(\lambda \zeta+c) \partial \rho$. This disc $f$ is extremal. 
Let $\mathscr{D}^{M}$ denote the family of extremal discs in $A^{M}$ described by Proposition 4. Given that extremal discs are always stationary, it follows that $\mathscr{D}^{M}$ contains all small extremal discs attached to $M$. It is sufficient to consider this family of discs to prove our result.

The dimension of $\mathscr{D}^{M}$ is at most 15 . In fact, discs in $\mathscr{D}^{M}$ are described by the parameters $\lambda, c, w_{0}, y_{0}, v$ that span $\mathbb{C}^{2} \times \mathbb{R}^{2} \times \mathbb{C}^{2} \times \mathbb{R}^{2} \times \mathbb{C}^{2}$. However, the dependence of $f$ on the parameters $\lambda, c$ is not injective: if the vector $(\lambda, c)$ determines the lift of a disc $f$ in $\mathscr{D}^{M}$, then for any real $k$, the vectors $(k \lambda, k c)$ determine lifts of $f$ as well.

In order to state the next lemma (see [Tumanov 2001]), which is the starting point for our proof, we introduce a few objects and some notation. We denote by $[\lambda, c]$ the classes of pairs $(\lambda, c)$ that are equivalent up to a proportionality factor. We set

$$
B=\left[\begin{array}{cc}
\operatorname{Re}\left(\lambda_{1} \zeta+c_{1}\right) & 0 \\
0 & \operatorname{Re}\left(\lambda_{2} \zeta+c_{2}\right)
\end{array}\right] .
$$

Condition (3) implies the existence of unique $a_{i} \in R^{+}$and $b_{i} \in \mathbb{C}$ such that $\left|b_{i}\right|>1$ and $\operatorname{Re}\left(\lambda_{i} \zeta+c_{i}\right)=a_{i}\left(\zeta-b_{i}\right)\left(\bar{\zeta}-\bar{b}_{i}\right)$ for $|\zeta|=1$ and $i=1,2$. Let $a=\left(a_{1}, a_{2}\right)$ and $b=\left(b_{1}, b_{2}\right)$. For $k \in R^{+}$we denote the equivalence class of pairs $(k a, b)$ by $[a, b]^{*}$. The correspondence between the classes $[\lambda, c]$ and $[a, b]^{*}$ is bijective. We define

$$
P=\left[\begin{array}{cc}
P_{1} & 0 \\
0 & P_{2}
\end{array}\right]
$$

where $P_{i}=\sqrt{a_{i}}\left(\zeta-b_{i}\right)$ and we denote the conjugate of $P$ by $P^{*}$. We set

$$
\nabla g_{i}=\left[\begin{array}{c}
\frac{\partial g_{i}}{\partial w_{1}} \\
\frac{\partial g_{i}}{\partial w_{2}}
\end{array}\right] .
$$

Finally, we introduce the Cauchy-type operator

$$
K(f)(\zeta)=\frac{1}{2 \pi i} \int_{b \Delta} \frac{f(\xi)}{\xi-\zeta} d \xi
$$

for $f$ in $C^{0}(b \Delta)$ where, for $|\zeta|=1$, we understand $K(f)(\zeta)$ to be the inner boundary value of the expression. We set $K_{0}(f)(\zeta)=K(f)(\zeta)-K(f)(0)$ and write $K_{0}\left(f_{1}, f_{2}\right)$ for $\left(K_{0}\left(f_{1}\right), K_{0}\left(f_{2}\right)\right)$.

Let $f(\zeta)=(z(\zeta), w(\zeta))$ be a disc in $\mathscr{A}^{M}\left(w_{0}^{\prime}\right)$ and define $u(\zeta)$ by $u(\zeta)=w(\zeta) / \zeta$ (recall that $w(0)=0)$. 
Lemma 5. The disc $f(\zeta)$ is extremal with lift determined by $(\lambda, c)$ if and only if (4) $u(\zeta)=P^{-1}(\zeta) P(0) u(0)$

$$
-P^{-1}(\zeta) K_{0}\left(\bar{\xi} P^{*-1} P_{1}^{*} P_{1} \overline{\nabla g_{1}}+\bar{\xi} P^{*-1} P_{2}^{*} P_{2} \overline{\nabla g_{2}}\right)(\zeta)
$$

on $b \Delta$.

(With an abuse of notation, we use $\xi$ in the argument of $K_{0}$ to indicate the identity function.)

Proof. By Definition 2 and Proposition 4 the disc $f$ is extremal with lift $f^{*}=$ $\operatorname{Re}(\lambda \zeta+c) \partial \rho$ if and only if $\zeta \operatorname{Re}(\lambda \zeta+c) h_{w}(w(\zeta))$, defined on $b \Delta$, extends to a continuous function on $\bar{\Delta}$ holomorphic on $\Delta$.

Given the above notations, and equation (2), we have

$$
\zeta \operatorname{Re}(\lambda \zeta+c) h_{w}(w(\zeta))=P P^{*} \bar{u}+\zeta P_{1}^{*} P_{1} \nabla g_{1}+\zeta P_{2}^{*} P_{2} \nabla g_{2}
$$

for $|\zeta|=1$.

Therefore the function $\zeta \operatorname{Re}(\lambda \zeta+c) h_{w}(w(\zeta))$ extends to a holomorphic function on $\Delta$ if and only if

$$
K_{0}\left(\overline{P P^{*} \bar{u}+\xi P_{1}^{*} P_{1} \nabla g_{1}+\xi P_{2}^{*} P_{2} \nabla g_{2}}\right)(\zeta)=0 .
$$

The matrix $P^{*-1}$ is anti-holomorphic, and defined in $\bar{\Delta}$ as $\left|b_{i}\right|>1$. Therefore the last equality becomes

$$
K_{0}(P u)(\zeta)=-K_{0}\left(\bar{\xi} P^{*-1} P_{1} P_{1}^{*} \nabla g_{1}+\bar{\xi} P^{*-1} P_{2} P_{2}^{*} \nabla g_{2}\right)
$$

and yields the conclusion, given that $P u(\zeta)=P(0) u(0)+K_{0}(P u(\zeta))$, since $P u(\zeta)$ is holomorphic on $\Delta$

A straightforward rescaling of coordinates shows that $Q_{\sigma}$ is biholomorphically equivalent to $Q_{1}$ if $\sigma \neq 0$, therefore we restrict our consideration to this last manifold. We denote by $D^{M}\left(w_{0}^{\prime}\right)$ the set

$$
\left\{(z(\zeta), w(\zeta)) \in D^{M}: w(0)=0, y(0)=0, w^{\prime}(0)=w_{0}^{\prime}\right\} .
$$

Theorem 1 follows from the following proposition.

Proposition 6. There exists $\epsilon>0$ such that if $f \in \mathscr{D}^{Q_{1}}\left(w_{0}^{\prime}\right)$ is extremal with respect to a class $[\lambda, c]$, where $\left|w_{0}^{\prime}\right|<\epsilon$, then $f$ is extremal only with respect to $[\lambda, c]$.

Proof. Our manifold $Q_{1}$ is defined by (1) with $\sigma=1$, so (4) becomes

$$
\begin{aligned}
& u_{1}(\zeta)=-\frac{b_{1}}{\zeta-b_{1}} u_{1}(0)-\frac{1}{\sqrt{a_{1}}\left(\zeta-b_{1}\right)} K_{0}\left(\bar{\xi} P_{1} u_{2} \bar{u}_{1}\right)(\zeta) \\
& u_{2}(\zeta)=-\frac{b_{2}}{\zeta-b_{2}} u_{2}(0)-\frac{1}{\sqrt{a_{2}}\left(\zeta-b_{2}\right)} K_{0}\left(\frac{1}{2} \xi P_{2}^{*-1} P_{1}^{*} P_{1} u_{1}^{2}\right)(\zeta)
\end{aligned}
$$


We set $u(0)=t v$, for $t \in \mathbb{R}^{+}$and $v \in \mathbb{C}^{2}$ with $|v|=1$. Then $u$ is a function $u(\zeta, a, b, v, t)$ holomorphic in $\zeta$ for $|\zeta|<1$, for every choice of the parameters $a, b, v$ and $t$ (recall that $a_{i}>0$ and $\left|b_{i}\right|>1$ ). We determine its Taylor expansion up to the second order in $t$ at 0 , starting from (5). If $t=0$, then (5) gives equations of type $u=O_{2}(u)$ where $O_{2}$ denotes terms vanishing at 0 of order at least 2 in $u$. This system has the unique solution

$$
u(\zeta, a, b, v, 0)=0 .
$$

We differentiate (5) twice with respect to $t$ and evaluate the resulting expressions at $t=0$; denoting $(\partial u / \partial t)(\zeta, a, b, v, 0)$ by $\dot{u}(0)$, we obtain

$$
\dot{u}_{1}(0)=-\frac{b_{1}}{\zeta-b_{1}} v_{1}, \quad \dot{u}_{2}(0)=-\frac{b_{2}}{\zeta-b_{2}} v_{2}
$$

and

$$
\ddot{u}_{1}(0)=-\frac{1}{\sqrt{a_{1}}\left(\zeta-b_{1}\right)} K_{0}\left(\bar{\xi} P_{1} 2 \dot{u}_{2}(0) \dot{\bar{u}}_{1}(0)\right)(\zeta),
$$

$$
\ddot{u}_{2}(0)=-\frac{1}{2 \sqrt{a_{2}}\left(\zeta-b_{2}\right)} K_{0}\left(\xi P_{2}^{*-1} P_{1}^{*} P_{1} 2 \dot{u}_{1}^{2}(0)\right)(\zeta)
$$

We evaluate $K_{0}$ in (8) and obtain

$$
\begin{aligned}
& \ddot{u}_{1}(0)=-2 v_{2} \bar{v}_{1}\left(\frac{\bar{b}_{1}\left(b_{2}-b_{1}\right)}{1-b_{2} \bar{b}_{1}}\right) \frac{\zeta}{\left(\zeta-b_{2}\right)\left(\zeta-b_{1}\right)}, \\
& \ddot{u}_{2}(0)=-\frac{a_{1}}{a_{2}} v_{1}^{2} b_{1}^{2}\left(\frac{1-\left|b_{1}\right|^{2}}{1-b_{1} \bar{b}_{2}}\right) \frac{\zeta}{\left(\zeta-b_{2}\right)\left(\zeta-b_{1}\right)} .
\end{aligned}
$$

In conclusion, we fix $v$ and consider $t$ small enough that we can neglect terms of order greater than 2 in the expansion of $u$. For a given solution $u$ of (4), there exists a unique pair $\left(b_{1}, b_{2}\right)$ satisfying (7), and there is a unique pair $\left(a_{1}, a_{2}\right)$, up to proportionality, that satisfies equations (9). This proves that $u(\zeta)$ and, with it, $w(\zeta)$, is determined by a unique class $[a, b]^{*}$.

Given the equivalence between classes $[a, b]^{*}$ and $[\lambda, \delta]$, we conclude that, for $w_{0}^{\prime}$ suitably small, an extremal disc $f=(z(\zeta), w(\zeta))$ attached to $Q_{1}$ with $w^{\prime}(0)=$ $w_{0}^{\prime}$ is extremal only with respect to one class $[\lambda, \delta]$.

\section{Acknowledgements}

We thank Professor Alexander Tumanov for frequent and invaluable discussions and Professor John D'Angelo for many useful remarks. 


\title{
References
}

[Boggess 1991] A. Boggess, CR manifolds and the tangential Cauchy-Riemann complex, Studies in Advanced Mathematics, CRC Press, Boca Raton, FL, 1991. MR 94e:32035 Zbl 0760.32001

[Lempert 1981] L. Lempert, "La métrique de Kobayashi et la représentation des domaines sur la boule”, Bull. Soc. Math. France 109:4 (1981), 427-474. MR 84d:32036 Zbl 0492.32025

[Sukhov and Tumanov 2001] A. Sukhov and A. Tumanov, "Stationary discs and geometry of CR manifolds of codimension two", Internat. J. Math. 12:8 (2001), 877-890. MR 2002i:32030 Zbl 01911906

[Tumanov 2001] A. Tumanov, "Extremal discs and the regularity of CR mappings in higher codimension”, Amer. J. Math. 123:3 (2001), 445-473. MR 2002f:32063 Zbl 0995.32024

Received May 13, 2004. Revised July 27, 2004.

\author{
ALBERTO SCALARI \\ CGC2 Citigroup CEnTre, 4TH Floor \\ CANADA SQUARE \\ CANARY WHARF \\ LONDON E14 5LB \\ UNITED KINGDOM \\ alberto.scalari@citigroup.com
}

